\title{
ARTí́CULO
}

\section{Influencia de las variables fisicoquímicas en la estructura de tallas y distribución de Meoma ventricosa grandis (Echinodermata: Brissidae) dentro del canal Boca Chica, Acapulco, México}

Influence of physicochemical variables in the size structure and distribution of Meoma ventricosa grandis (Echinodermata: Brissidae) within the Boca Chica Channel, Acapulco, Mexico

\author{
Miguel A. Torres-Martínez ${ }^{1 *}$, Francisco A. Solís-Marín², \\ Alfredo Laguarda-Figueras ${ }^{2}$ y Francisco Sour-Tovar ${ }^{3}$
}

${ }^{1}$ Instituto de Geología, Departamento de Paleontología, Universidad Nacional Autónoma de México, Ciudad Universitaria, 04510 México, CDMX, México.*miguelatm@geologia.unam.mx

${ }^{2}$ Instituto de Ciencias del Mar y Limnología, Laboratorio de Sistemática y Ecología de Equinodermos, Universidad Nacional Autónoma de México, Apdo. post. 70-305, México, CDMX, 04510, México

${ }^{3}$ Museo de Paleontología, Departamento de Biología Evolutiva, Facultad de Ciencias, Universidad Nacional Autónoma de México, Ciudad Universitaria, 04510 México, CDMX, México

\begin{abstract}
In this study we examine different physicochemical variables (sediment texture, temperature, salinity, dissolved oxygen, turbidity, nitrite, nitrate, ammonium, phosphate, organic matter of marine snow, organic matter of sediment and organic matter of digestive tract) and their influence on the size structure and distribution of Meoma ventricosa grandis. The study was conducted in 3 workstations (E1: La Playa, E2: El Jardín, E3: Palmitas) located across the Boca Chica Channel, Acapulco, Mexico, during 2006 to 2007. The results of the measurements of the body size showed a higher percentage of adults in workstation 1 and 2 (13 and $5 \%$, respectively); however, in workstation 3 the percentage was of $51 \%$. The variance pointed a significance in the size of the individuals from the workstation Palmitas, showing the largest specimens throughout the study. The sediment texture in workstations La Playa and El Jardín was characterized by asymmetric clasts of coarse sand and gravel of different sizes, with a sediment layer with a depth of layer of $20-30 \mathrm{~cm}$. Unlike workstation of Palmitas wich was distinguished by almost symmetrical gravel clasts, similar in size and with depth of $50 \mathrm{~cm}$; in all workstations juveniles were buried between 10 and $20 \mathrm{~cm}$ and adults between 15 and $40 \mathrm{~cm}$ of depth. The gonadal index was asynchronous, presenting the highest reproductive peaks in Palmitas. Regression Trees analysis established that the organic matter deposited in the sediment acts as the primary physicochemical variable in the distribution of the echinoid within the channel, locating until 6,571 individuals in direct relation to it. The largest number of specimens (11,330) was obtained in the presence of organic matter, nitrates and phosphates. This is the first time that genus Meoma is related to a specific substrate according to the body size.
\end{abstract}

Key words: Meoma ventricosa grandis, physicochemical variables, size structure, distribution, Acapulco

Resumen.- En este trabajo se examinaron diferentes variables fisicoquímicas (textura del sedimento, temperatura, salinidad, oxígeno disuelto, turbidez, nitrito, nitrato, amonio, fosfato, materia orgánica de nieve marina, materia orgánica del sedimento y materia orgánica del tracto digestivo) y su influencia en la estructura de tallas y la distribución de Meoma ventricosa grandis. El estudio fue realizado en 3 estaciones de trabajo (E1: La Playa, E2: El Jardín, E3: Palmitas) localizadas a través del canal Boca Chica, Acapulco, México, durante 2006 y 2007. Los resultados de las mediciones del tamaño corporal mostraron un menor porcentaje de individuos adultos en las estaciones 1 y 2 (13 y 5\%, respectivamente), por el contrario en la estación 3 el porcentaje fue de 51\%. El análisis de varianza señaló una diferencia significativa en el tamaño de los individuos de la estación Palmitas con respecto a las otras dos, presentando los ejemplares más grandes a través de todo el estudio. La textura del sedimento en la estación 1, La Playa y en la estación 2, El Jardín se caracterizó por clastos asimétricos de arena gruesa y grava, de diversos tamaños, con una profundidad de la capa de sedimentos de 20 a $30 \mathrm{~cm}$, en contraste con la estación 3 , Palmitas, la cual presentó clastos casi simétricos de arena gruesa, de tamaño similar, con profundidad de $50 \mathrm{~cm}$; en todas las estaciones los individuos juveniles estaban enterrados entre los 10 y $20 \mathrm{~cm}$ de profundidad y los adultos entre 15 y $40 \mathrm{~cm}$. El índice gonádico fue de tipo asincrónico durante el estudio, presentando los picos reproductivos más altos en Palmitas. El análisis de árboles de regresión estableció que la materia orgánica consumida por los individuos actúa como la principal variable fisicoquímica que favorece la distribución de este equinoideo dentro del canal, localizando hasta 6,571 individuos en relación a este recurso. La mayor proporción de ejemplares $(11,330)$ se alcanzó en presencia de la materia orgánica, los nitratos y los fosfatos. Se registra por primera vez para el género Meoma la existencia de una selección específica del tipo de sustrato dependiente principalmente de su talla corporal.

Palabras clave: Meoma ventricosa grandis, variables fisicoquímicas, estructura de tallas, distribución, Acapulco 


\section{INTRODUCCIÓN}

La relación que existe entre un organismo bentónico y un ambiente en particular se ha documentado para diferentes tipos de invertebrados (Méndez-Ubach et al. 1986, Snelgrove \& Butman 1994, Pinedo et al. 2000, Iken et al. 2001, Gray 2002, Challener et al. 2009, Boos et al. 2010, Aslan-Cihangir \& Pancucci-Papadopoulou 2012). Esta afinidad generalmente se asocia únicamente a la cantidad de materia orgánica presente en el medio o con la textura del sedimento, pero es evidente que existen otras variables ambientales que determinan los patrones de distribución espacial de un animal dentro de su hábitat (Freeman \& Rogers 2003) y muchos rasgos de las especies bentónicas, como tasas de supervivencia o reproducción, niveles tróficos, morfologías o desarrollo de adaptaciones particulares (Pulliam 2000, Ramírez-Llodra et al. 2002, Guzmán \& Guevara 2002). En este contexto se estudia a Meoma ventricosa grandis, equinoideo irregular que se distribuye ampliamente en aguas tropicales americanas del Océano Pacífico, desde Baja California Sur, México hasta Ecuador (Solís-Marín et al. 1997, 2013). Sobre esta subespecie, aún cuando es considerada como uno de los principales constituyentes de diferentes comunidades del litoral del Pacífico mexicano (Solís-Marín et al. 1997), existen pocas referencias que se limitan a análisis taxonómicos (Mortensen 1951, Solís-Marín et al. 1997, Torres-Martínez et al. 2008), listados paleontológicos (Buitrón-Sánchez et al. 1999) o trabajos preliminares sobre su biología (Torres-Martínez 2008, Torres-Martínez \& Solís-Marín 2010). Considerando la falta de información sobre este equinoideo, en el presente trabajo se analiza la distribución espacial de $M$. ventricosa grandis en el canal Boca Chica, Acapulco, se evalúa su relación con diferentes variables físicas y químicas del ambiente y se registra por primera vez el ciclo reproductivo anual de la subespecie y su afinidad con las estructuras de tallas corporales encontradas en cada parche poblacional.

\section{Materiales Y MÉTODOS}

\section{Área de ESTUdio}

El canal Boca Chica se localiza al sur de la península de Las Playas en el municipio de Acapulco de Juárez y al norte de la isla Roqueta, Guerrero, México (16 $49,81^{\circ}$ y $16^{\circ} 49,45^{\prime} \mathrm{N}$; 99 54,00' y 99 $\left.55,00^{\circ} \mathrm{O}\right)$. Tanto el canal Boca Chica como el canal de la Yerbabuena comunican a la bahía de Acapulco con el océano y presentan marea mixta semidiurna de $0,65 \mathrm{~m}$ de amplitud media (http://www.mareografico.unam.mx/). Dentro del canal se ubicaron las 3 estaciones de trabajo: estación 1 (La Playa) a 1649,81’N; 9954,06’O, estación 2 (El Jardín) a 16²4,44’ N; 9954,97'O, y estación 3 (Palmitas) situada a $16^{\circ} 49,44^{\prime} \mathrm{N}$; $99^{\circ} 54,73^{\prime} \mathrm{O}$ (Fig. 1). En la Tabla 1 se muestra la profundidad en la columna de agua del suelo marino, la profundidad de cada capa de sedimento y las características sedimentarias generales de cada estación. Meoma ventricosa grandis se encontró asociado a otros invertebrados bentónicos como poliquetos, bivalvos, gasterópodos y otros equinodermos.

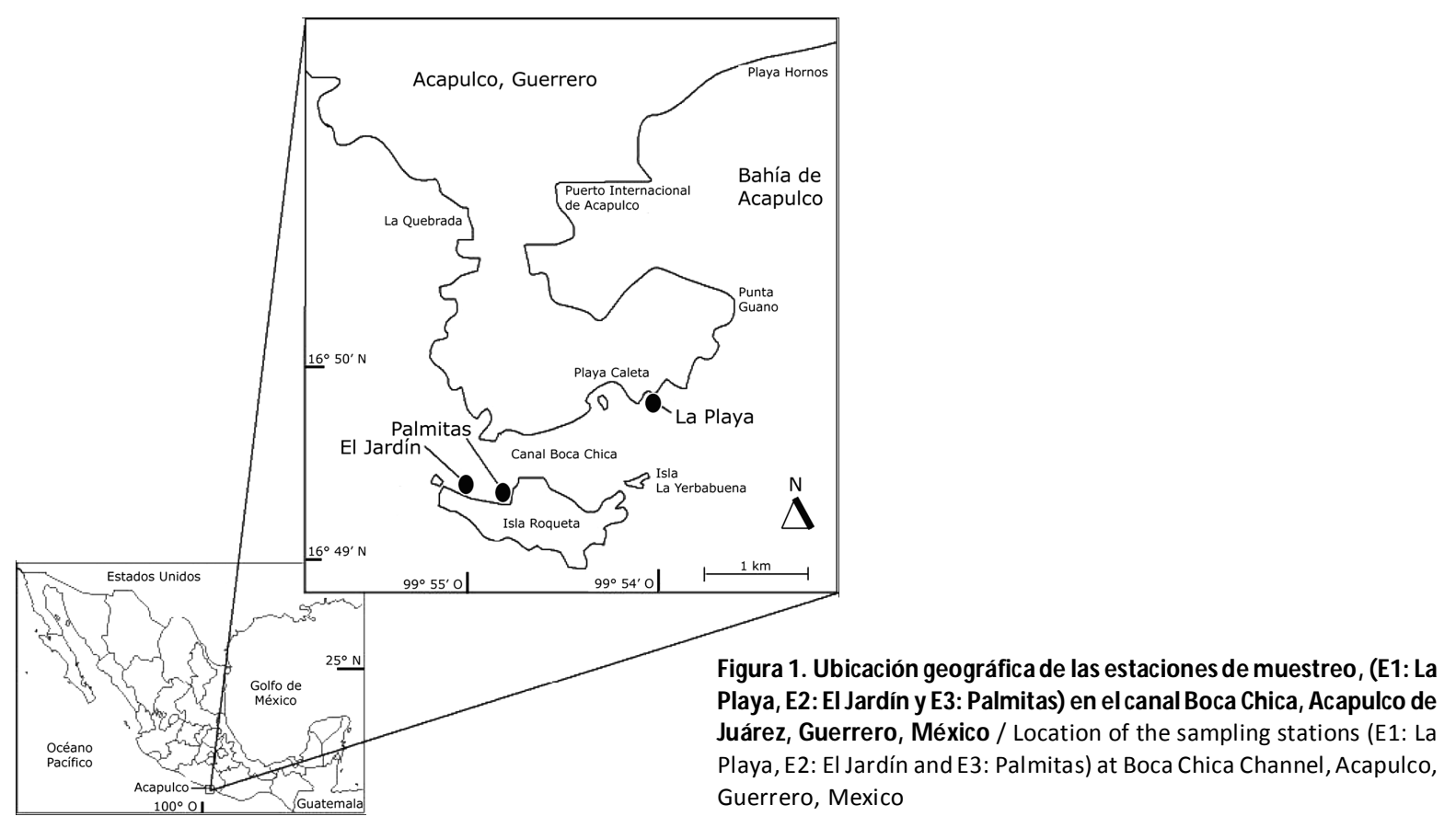


Tabla 1. Características físicas de las 3 estaciones muestreadas (E1: La Playa, E2: El Jardín y E3: Palmitas). TS - textura del sedimento; PF profundidad; PCS - profundidad de la capa de sedimento / Physical features from 3 sampled stations (E1: La Playa, E2: El Jardín and E3: Palmitas). TS - texture of sediment; PF - depth; PCS - depth of the sediment layer

\begin{tabular}{cccc}
\hline Estación & TS & PF (m) & PCS (cm) \\
\hline E1 & grava y arena, con gran cantidad & 11,2 & 30 \\
E2 & de bioclastos & 12 & 20 \\
E3 & arena muy gruesa sin bioclastos & 7,5 & 50 \\
\hline
\end{tabular}

\section{Trabajo de campo}

El esfuerzo de muestreo se llevó a cabo en 11 temporadas de campo, una cada mes entre marzo 2006 y marzo 2007, cada una abarcando 3 días de trabajo efectivo. Los organismos, las muestras de agua, de sedimento y de nieve marina se recolectaron mediante buceo SCUBA.

Para realizar la recolecta de Meoma ventricosa grandis se localizaron sus parches poblacionales mediante la observación de oquedades de sedimento removido. Una vez ubicados, fueron desenterrados manualmente, con un Vernier se tomaron las medidas morfológicas de mayor longitud (TL), ancho (W) y alto de la testa $(\mathrm{H})$ para obtener el tamaño total aproximado de cada uno de los individuos. La profundidad del sedimento a la que se encontraba enterrado cada espécimen fue medida empleando una regla de $30 \mathrm{~cm}$ de largo. Dado que se buscaba perturbar lo menos posible el área de recolecta de los ejemplares extraídos en cada estación, 5 fueron medidos in situ para ser regresados al medio y el resto se recolectó en una cubeta con agua de mar para ser medidos y diseccionados posteriormente en el laboratorio. Durante cada salida de campo, se obtuvo el peso corporal in vivo de cada individuo y posteriormente cada ejemplar fue diseccionado para extraer su tracto digestivo y gónadas. En el laboratorio, las gónadas fueron pesadas, etiquetadas y fijadas en formol al 7\%, conservándose en alcohol al 70\%. Con los datos del peso del individuo y de sus gónadas se calculó el índice gonádico (IG) mediante la fórmula IG = (masa de la gónada $/$ masa corporal) $\mathrm{x} 100$. Este índice identifica los periodos reproductivos de un organismo, dado que la maduración germinal y el desove coinciden con el peso máximo gonadal (Williamson \& Steinberg 2002). Además, los ejemplares adultos fueron diferenciados de los juveniles por presentar una longitud corporal igual o mayor a $80 \mathrm{~mm}$. La madurez reproductiva (etapas III y IV) fue corroborada mediante la técnica histológica de hematoxilinaeosina.
La obtención de los datos de temperatura, salinidad, turbidez y oxígeno disuelto presentes en el contacto sedimento-agua se realizó utilizando un HYDROLAB Data Sonde 3-HL002008. Para el análisis granulométrico se recolectaron 200 g de sedimento en cada una de las zonas de estudio.

El aporte de materia orgánica (MO) que se deposita mensualmente en las 3 regiones se determinó mediante el uso de trampas de nieve marina (5 por estación). Para identificar la cantidad de nutrientes se tomaron muestras de agua de $5 \mathrm{ml}$ para el análisis de nitrato, nitrito y fosfato y $30 \mathrm{ml}$ para el amonio. Para llevar a cabo el análisis de MO se tomaron muestras de 1 $\mathrm{kg}$ de sedimento para ser procesadas junto con la nieve marina y los tractos digestivos.

Todo el material fue etiquetado y conservado bajo refrigeración hasta ser analizado posteriormente. Los datos de las diferentes variables (temperatura, salinidad, oxígeno disuelto, turbidez, nitrito, nitrato, amonio, fosfato, MO de trampas, MO del sedimento y MO del tracto digestivo) fueron registrados mensualmente durante el año de estudio.

\section{Trabajo de laboratorio}

El trabajo de laboratorio abarcó el estudio de la histología de las gónadas de los organismos, el análisis textural y la cuantificación de nutrientes y MO de las muestras correspondientes.

Para comprobar la madurez reproductiva de los ejemplares se realizó la técnica histológica de hematoxilina-eosina, método que consiste en la deshidratación de las gónadas utilizando diferentes grados de alcohol, lavadas posteriormente con xilol y colocadas en parafina para realizar secciones seriales de 6 $\mu \mathrm{m}$. Posteriormente se retira la parafina, se tiñe con hematoxilinaeosina y los cortes histológicos son montados en Bálsamo de Canadá (INECC 2009).

El análisis granulométrico de cada estación se realizó utilizando un agitador Ro-Tap Testing Sieve Shaker, pasando por una serie de tamices U.S. Standard de $-3 \phi$ a $4 \phi$ (Folk 1980). El material de cada tamiz fue pesado para obtener los percentiles y establecer las características granulométricas del sedimento. El tamaño gráfico promedio o tamaño de grano $\left(\mathrm{M}_{\mathrm{z}}\right)$ y la desviación estándar gráfica inclusiva o clasificación $\left(\sigma_{\mathrm{I}}\right)$ se representarán con el número $\phi$ y el grado de asimetría gráfica inclusiva $\left(\mathrm{Sk}_{\mathrm{I}}\right)$ se distinguirá como el rango de asimetría de los clastos. El material sedimentario de las 3 estaciones se relacionó con rangos texturales específicos de la clasificación de Folk: $\mathrm{M}_{\mathrm{z}}=$ Arena muy gruesa: $-1 \phi$ a $0 \phi(2$ a $1 \mathrm{~mm})$ y Gránulo: $-2 \phi$ a $-1 \phi(4 \mathrm{a} 2 \mathrm{~mm}) ; \sigma_{\mathrm{I}}=$ Moderadamente clasificado: $0,71 \phi \mathrm{a}$ $1,00 \phi$ y Bien clasificado: $0,35 \phi$ a $0,50 \phi ; \mathrm{Sk}_{\mathrm{I}}=$ Muy 
asimétrico hacia gruesos: -0,30 a -1,00 y Asimétrico hacia finos: $+0,30$ a $+0,10$.

Las variables químicas se analizaron en el Laboratorio de Fisicoquímica del Instituto de Ciencias del Mar y Limnología de la U.N.A.M., México. Los resultados de los nutrientes se obtuvieron de las muestras de agua por medio de espectrofotometría, técnica que permite determinar la concentración de un compuesto en solución mediante radiaciones electromagnéticas emitidas en un espectrofotómetro (Díaz et al. 2000). Los datos de MO del sedimento, de la nieve marina y de los tractos digestivos se obtuvieron con el método de titulación manual, que se reconoce como el procedimiento estándar para la obtención de MO en suelos y sedimentos, donde un porcentaje de la muestra de sedimento se oxida con dicromato de potasio $\left(\mathrm{H}_{2} \mathrm{SO}_{4}\right)$, posteriormente el exceso de dicromato se titula con una solución de sulfato ferroso hasta que se produce un cambio de coloración de azul a verde, funcionando la difenilamina sulfonato de sodio como indicador (Garay et al. 2003). El porcentaje de MO en trampas de nieve marina, sedimento y tractos digestivos se determinó de acuerdo a la clasificación de porcentajes de MO de Moreno (1978).

\section{Análisis de datos}

El estudio del tamaño de los individuos de M. ventricosa grandis requirió reconocer la talla aproximada de cada organismo en $\mathrm{cm}^{3}$ a partir de las medidas corporales descritas previamente; mientras que para el índice gonádico se emplearon los resultados del índice obtenidos mensualmente.

Para establecer las diferencias del tamaño de los individuos y del índice gonádico entre las 3 estaciones, se realizó en cada caso un análisis de varianza (ANOVA), con su respectiva prueba de Fisher $* P<0,05 * * P<0,025 * * * P<0,001$. Los análisis de varianza de ambas variables se obtuvieron mediante el programa estadístico StatView - SAS Institute Inc., versión 5.

\section{ANÁlisis de VARIables}

Los datos de cantidad de individuos de $M$. ventricosa grandis registrados por estación junto con los resultados de las diferentes variables, fueron comparados mediante el análisis de árboles de regresión (RT, Regression Trees). Este procedimiento se utiliza como un modelo de aproximación que permite establecer cuáles de las diferentes variables independientes (predictoras) influyen principalmente sobre una variable dependiente (de respuesta), repercutiendo directamente en su comportamiento (Barber et al. 2001, Díaz \& Correa 2013). El análisis definió qué variables son las más significativas para que $M$. ventricosa grandis se pueda encontrar dentro del canal.
Los datos de las 3 estaciones se agruparon en una matriz con 12 variables independientes: textura del sedimento junto con 4 variables físicas: temperatura, salinidad, oxígeno disuelto y turbidez, y 7 variables químicas: nitrito, nitrato, amonio, fosfato, MO del sedimento, MO de trampas de nieve marina y MO del tracto digestivo, siendo la cantidad de individuos de $M$. ventricosa grandis presentes en cada estación la variable dependiente. El análisis RT se llevó a cabo por medio del programa estadístico S-Plus (Insightful Corp., Redmond, WA, USA) versión 2000 Professional.

\section{Resultados}

\section{TAMAÑo CORPORAL Y NÚMERO DE ORGANISMOS}

Se contaron 311 individuos entre las 3 estaciones, diferenciando entre adultos y juveniles. Esta información permitió definir la distribución por talla de los ejemplares de M. ventricosa grandis dentro del canal Boca Chica. En la Tabla 2 se observa que la estación 1 (La Playa) presentó 13\% de especímenes adultos, mientras que la estación 2 (El Jardín) tuvo 5\%. Por el contrario, en la estación 3 (Palmitas) se detectó un porcentaje de $51 \%$ de individuos adultos. Para corroborar esta disimilitud entre los valores de tamaño de los erizos entre estaciones se realizó el análisis de varianza correspondiente. Se comprobó una diferencia en la estructura de tallas entre los organismos encontrados en cada estación (Tabla 3), resaltando el valor significativo de la estación 3 (Palmitas) en comparación con las otras dos, las cuales presentaron los ejemplares de menor tamaño.

\section{Características del sustrato}

El análisis granulométrico de las muestras de sedimento de las 3 estaciones estableció las condiciones texturales bajo las que se desarrollan cada uno de los parches poblacionales de $M$. ventricosa grandis dentro del canal. De acuerdo a Folk (1980) el sedimento presentará diferentes límites texturales con los cuáles será posible caracterizar la granulometría de un sustrato en particular.

En la Tabla 4 se aprecian los resultados numéricos de textura del sedimento derivados de los diferentes percentiles granulométricos. De acuerdo a la clasificación de Folk (1980) en la estación 1 (La Playa) predominaron las gravas gruesas, moderadamente clasificadas y muy asimétricas. La estación 2 (El Jardín) se caracterizó por clastos de arena muy gruesa con tendencia hacia gravas, moderadamente clasificadas y muy asimétricas. Finalmente, la estación 3 (Palmitas) se distinguió por clastos de arena muy gruesa, que al contrario de las otras 2 estaciones estuvo bien clasificada, con asimetría hacia finos pero con tendencia a ser simétricos. 
Tabla 2. Número de individuos encontrados en cada estación estudiada (E1: La Playa, E2: El Jardín y E3: Palmitas) entre marzo 2006 a marzo 2007 / Number of individuals found in each workstation sampled (E1: La Playa, E2: El Jardín and E3: Palmitas) between March 2006 to March 2007

\begin{tabular}{ccccccc}
\hline & \multicolumn{2}{c}{ La Playa (E1) } & \multicolumn{2}{c}{ E1 Jardín (E2) } & \multicolumn{2}{c}{ Palmitas (E3) } \\
\cline { 2 - 7 } & \multirow{2}{*}{ Juveniles } & Adultos & Juveniles & Adultos & Juveniles & Adultos \\
\hline Marzo & 12 & 0 & 22 & 4 & 2 & 2 \\
Abril & 12 & 0 & 8 & 1 & 0 & 5 \\
Mayo & 15 & 1 & 18 & 0 & 4 & 7 \\
Julio & 8 & 2 & 9 & 1 & 7 & 3 \\
Agosto & 8 & 2 & 10 & 0 & 0 & 0 \\
Septiembre & 4 & 4 & 6 & 0 & 3 & 3 \\
Octubre & 8 & 2 & 10 & 0 & 0 & 1 \\
Noviembre & 11 & 0 & 10 & 0 & 3 & 5 \\
Enero & 8 & 2 & 10 & 0 & 1 & 1 \\
Febrero & 9 & 1 & 10 & 0 & 10 & 0 \\
Marzo & 9 & 1 & 11 & 0 & 0 & 4 \\
\hline$\Sigma$ & 104 & 15 & 124 & 6 & 30 & 31 \\
\hline$\%$ & 87 & 13 & 95 & 5 & 49 & 51 \\
\hline
\end{tabular}

Tabla 3. Promedio anual (2006-2007) del tamaño corporal de M eoma ventricosa grandis entre las estaciones, E1: La Playa, E2: El Jardín y E3: Palmitas. *P $<0,05 * * P<0,025 * * * P<0,001 /$ Yearly average (2006-2007) of body size of M eoma ventricosa grandis between the workstations, E1: La Playa, E2: El Jardín and E3: Palmitas. $* \mathrm{P}<0.05 * * \mathrm{P}<0.025 * * * \mathrm{P}<0.001$

\begin{tabular}{lcccc}
\hline Estaciones & $\begin{array}{c}\text { Suma de } \\
\text { cuadrados }\end{array}$ & $\begin{array}{c}\text { Cuadrado } \\
\text { medio }\end{array}$ & F & $P$ \\
\hline E1 y E2 & & & & $<0,025$ \\
E1 y E3 & 681485,699 & 340742,849 & 53,448 & $\begin{array}{l}<0,001 \\
\text { E2 y E3 }\end{array}$ \\
\hline
\end{tabular}

Tabla 4. Medidas de uniformidad del sedimento obtenidas de las 3 estaciones(E1: La Playa, E2: El Jardín y E3: Palmitas) del canal Boca Chica, Acapulco de marzo 2006 a marzo 2007. $M_{z}(\phi)$ - tamaño gráfico promedio (tamaño de grano); $\sigma_{1}(\phi)$ - desviación estándar gráfica inclusiva (clasificación); $\mathbf{S k}$ - grado de asimetría gráfica inclusiva (asimetría) / Uniformity mesurements of sediment obtained from 3 stations (E1: La Playa, E2: El Jardín and E3: Palmitas) from Boca Chica cannel, Acapulco of March 2006 to March 2007. $M_{z}(\phi)$ - graphic mean (grain size); $\sigma_{1}(\phi)$ - inclusive graphic standard deviation (classification); $S k_{1}$ - inclusive graphic skewness (asymmetry)

\begin{tabular}{|c|c|c|c|c|c|c|}
\hline \multirow{3}{*}{$\mathrm{M}_{\mathrm{Z}}(\phi)$} & \multirow{2}{*}{\multicolumn{2}{|c|}{$\frac{\text { La Playa (E1) }}{\text { Promedio y D.E. }}$}} & \multirow{2}{*}{\multicolumn{2}{|c|}{$\begin{array}{c}\text { E1 Jardín (E2) } \\
\text { Promedio y D.E. }\end{array}$}} & \multirow{2}{*}{\multicolumn{2}{|c|}{$\frac{\text { Palmitas (E3) }}{\text { Promedio y D.E. }}$}} \\
\hline & & & & & & \\
\hline & $-1,12$ & $\pm 0,56$ & $-0,93$ & $\pm 0,63$ & $-0,63$ & $\pm 0,19$ \\
\hline$\sigma_{I}(\phi)$ & 0,795 & $\pm 0,288$ & 0,838 & $\pm 0,300$ & 0,474 & $\pm 0,077$ \\
\hline $\mathrm{Sk}_{\mathrm{I}}$ & $-0,492$ & $\pm 1,815$ & $-1,132$ & $\pm 5,735$ & 0,110 & $\pm 0,099$ \\
\hline
\end{tabular}


Hay que resaltar que los ejemplares juveniles se encontraban enterrados en un rango de 10 a $20 \mathrm{~cm}$ de profundidad en el sustrato, mientras que los adultos entre los 15 a $40 \mathrm{~cm}$.

\section{VARIABLES FISICOQUÍMICAS}

La información obtenida en campo sobre la temperatura, salinidad, turbidez y oxígeno disuelto, junto con los datos generados en el laboratorio de las diferentes variables químicas permitieron conocer el comportamiento de las diferentes variables independientes dentro del canal. En la Tabla 5 se aprecia que los factores fisicoquímicos no variaron significativamente entre las 3 estaciones a lo largo del año. Al relacionar entre sí los datos del aporte de materia orgánica de trampas de nieve marina, del sedimento y del tracto digestivo con la clasificación de Moreno (1978) se observaron algunas diferencias en el porcentaje de materia orgánica entre las 3 variables (Tabla 6).

Tabla 5. Media y desviación estándar de las variables independientes obtenidas en cada estación de muestreo (E1: La Playa, E2: El Jardín y E3: Palmitas) del canal Boca Chica, Acapulco de marzo 2006 a marzo 2007. T- temperatura ( $\left.{ }^{\circ} \mathrm{C}\right) ;$ Ssalinidad; OD- oxígeno disuelto ( $\left.\mathrm{mg} \mathrm{L}^{-1}\right)$; $\mathrm{Tz}^{-}$turbidez (NTU); $\mathrm{NO}_{2}$ - nitrito ( $\left.\mathrm{mg} \mathrm{L}^{-1}\right)$; $\mathrm{NO}_{3}$ - nitrato $\left(\mathrm{mg} \mathrm{L}^{-1}\right)$; $\mathrm{NH}_{4}$ - amonio (mg $\mathrm{L}^{-1}$ ); $\mathrm{PO}_{4}$ - fosfato ( $\mathrm{mg} \mathrm{L}^{-1}$ ); MOT- MO de trampas (\%); MOS- MO del sedimento (\%); MOD-MO del tracto digestivo (\%) / Mean and standard deviation of independent variables obtained at each sampling station (E1: La Playa, E2: El Jardín and E3: Palmitas) from Boca Chica Channel, Acapulco of March 2006 to March 2007. T- temperature $\left({ }^{\circ} \mathrm{C}\right)$; S- salinity; $\mathrm{OD}$ - dissolved oxygen ( $\left.\mathrm{mg} \mathrm{L}^{-1}\right)$; Tz- turbidity (NTU); $\mathrm{NO}_{2}$ - nitrite ( $\left.\mathrm{mg} \mathrm{L}^{-1}\right) ; \mathrm{NO}_{3}$ - nitrate ( $\left.\mathrm{mg} \mathrm{L}^{-1}\right) ; \mathrm{NH}_{4}$ - ammonium ( $\mathrm{mg} \mathrm{L}^{-1}$ ); $\mathrm{PO}_{4}$ - phosphate (mg L-1); MOT- OM of marine snow (\%); MOS- OM of sediment (\%); MOD- OM of digestive tract (\%)

\begin{tabular}{lllllll}
\hline & \multicolumn{2}{c}{ La Playa (E1) } & \multicolumn{2}{c}{ El Jardín (E2) } & \multicolumn{2}{c}{ Palmitas (E3) } \\
\cline { 2 - 7 } & \multicolumn{2}{c}{ Promedio y D.E } & \multicolumn{2}{c}{ Promedio y D.E } & \multicolumn{2}{c}{ Promedio y D.E } \\
\hline $\mathrm{T}$ & 28,7 & $\pm 1,94$ & 27,9 & $\pm 2,49$ & 28,7 & $\pm 1,75$ \\
$\mathrm{~S}$ & 33,000 & $\pm 1,59$ & 33,554 & $\pm 1,20$ & 33,345 & $\pm 1,24$ \\
$\mathrm{OD}$ & 6,138 & $\pm 0,54$ & 5,769 & $\pm 1,04$ & 6,606 & $\pm 0,66$ \\
$\mathrm{Tz}$ & 1,130 & $\pm 1,11$ & 0,654 & $\pm 1,28$ & 0,709 & $\pm 1,47$ \\
$\mathrm{NO}_{2}$ & 0,00583 & $\pm 0,00433$ & 0,00849 & $\pm 0,00756$ & 0,00911 & $\pm 0,01532$ \\
$\mathrm{NO}_{3}$ & 0,195 & $\pm 0,371$ & 0,166 & $\pm 0,244$ & 0,082 & $\pm 0,141$ \\
$\mathrm{NH}_{4}$ & 0,0064 & $\pm 0,0118$ & 0,0084 & $\pm 0,0218$ & 0,0117 & $\pm 0,0349$ \\
$\mathrm{PO}_{4}$ & 0,0244 & $\pm 0,0176$ & 0,0263 & $\pm 0,0188$ & 0,0542 & $\pm 0,0965$ \\
$\mathrm{MOT}$ & 1,84 & $\pm 0,73$ & 3,60 & $\pm 1,69$ & 2,29 & $\pm 1,07$ \\
$\mathrm{MOS}$ & 0,423 & $\pm 0,218$ & 0,505 & $\pm 0,166$ & 0,655 & $\pm 0,272$ \\
$\mathrm{MOD}$ & 1,50 & $\pm 0,85$ & 1,94 & $\pm 0,78$ & 1,56 & $\pm 0,76$ \\
\hline
\end{tabular}

Tabla 6. Valores de materia orgánica (MO) obtenidos en la 3 estaciones de muestreo (E1: La Playa, E2: El Jardín y E3: Palmitas) del canal Boca Chica, Acapulco (marzo 2006 a marzo 2007) y su interpretación de acuerdo a la clasificación de porcentajes de MO de Moreno (1978) / Organic material (OM) values obtained in the 3 sample stations (E1: La Playa, E2: El Jardín and E3: Palmitas) from Boca Chica channel, Acapulco (March 2006 to March 2007) and their interpretation according to the percentage classification of OM from Moreno (1978)

\begin{tabular}{|c|c|c|c|c|c|c|c|c|c|c|}
\hline \multirow{2}{*}{$\begin{array}{l}\% \text { de Materia orgánica } \\
\text { (MO) }\end{array}$} & \multirow[b]{2}{*}{ Interpretación } & \multicolumn{3}{|c|}{ MO de trampas } & \multicolumn{3}{|c|}{ MO de sedimento } & \multicolumn{3}{|c|}{ MO de tractos } \\
\hline & & E1 & E2 & E3 & E1 & E2 & E3 & E1 & E2 & E3 \\
\hline$<0,60$ & Extremadamente pobre & & & & - & - & & & & \\
\hline $0,60-1,20$ & Pobre & & & & & & $\bullet$ & & & \\
\hline $1,21-1,80$ & Medianamente pobre & & & & & & & - & & - \\
\hline $1,81-2,40$ & Mediano & - & & - & & & & & - & \\
\hline $2,41-3,00$ & Medianamente rico & & & & & & & & & \\
\hline $3,01-4,20$ & Rico & & - & & & & & & & \\
\hline$>4,20$ & Extremadamente rico & & & & & & & & & \\
\hline
\end{tabular}




\section{ÍNDICE GONÁDICO}

El análisis del IG detectó una diferencia significativa entre la actividad reproductiva de los individuos de la estación 3 (Palmitas) con los encontrados en las otras 2 estaciones (Tabla 7). Los resultados mensuales del IG revelaron que los picos de reproducción de $M$. ventricosa grandis fueron desiguales durante el año de estudio, al igual que fueron diferentes entre las estaciones. Por ejemplo de febrero a marzo se presentaron los picos reproductivos relativamente más altos en la estación 1 (La Playa), mientras que de abril a julio, septiembre, noviembre y enero los picos de la estación 3 (Palmitas) fueron los mayores. Si bien la estación 2 (El Jardín) presentó en todas las ocasiones valores bajos, en julio, septiembre, noviembre y enero superó ligeramente los valores de la estación 1 (La Playa) (Fig. 2).

El análisis del IG confirma la diferencia significativa de tamaño presente en los individuos de la estación 3 (Palmitas), ya que la relación del IG con la talla de los especímenes muestra picos reproductivos más altos en esta estación $(>0,001)$ comparados con la estación 1 (La Playa) y la 2 (El Jardín), los cuales no se diferenciaron significativamente entre ellos con excepción de febrero de 2007 (Tabla 8). Al presentar una conducta reproductiva aleatoria durante el año de estudio y no poderse asociar con un periodo de tiempo en particular, se señala que $M$. ventricosa grandis presenta un ciclo reproductivo de tipo asincrónico.
Tabla 7. Índice gonádico promedio anual de $M$. ventricosa grandis entre estaciones (E1: La Playa, E2: El Jardín y E3: Palmitas) del canal Boca Chica, Acapulco de 2006 a 2007. $* \mathrm{P}<0,05 * * \mathrm{P}<0,025 * * * \mathrm{P}<$ 0,001 / Yearly average of gonadal index of $M$. ventricosa grandis between stations (E1: La Playa, E2: El Jardín and E3: Palmitas) from Boca Chica Channel, Acapulco of 2006 to 2007. ${ }^{* P}<0.05 * * \mathrm{P}<0.025$ $* * * \mathrm{P}<0.001$

\begin{tabular}{ccccr}
\hline Estaciones & $\begin{array}{c}\text { Suma de } \\
\text { cuadrados }\end{array}$ & $\begin{array}{c}\text { Cuadrado } \\
\text { medio }\end{array}$ & F & $P$ \\
\hline E1 y E2 & & & & 0,274 \\
E1 y E3 & 89,296 & 44,648 & 30,016 & $\begin{array}{r}<0,001 \\
<0,001\end{array}$ \\
E2 y E3 & & & & \\
\hline
\end{tabular}

\section{ANÁlisis de ÁRBoles de REGRESIón (RT)}

El análisis de árboles de regresión (RT) aplicado a los datos de cantidad de individuos de $M$. ventricosa grandis frente a las 12 variables independientes estableció que el aporte de MO, el nitrato y el fosfato fueron los factores con mayor influencia en el número de individuos dentro del canal Boca Chica (Fig. $3)$. Los valores bajos de MO $(<1,07786)$ obtenidos en el área de estudio fueron relacionados con una abundancia de 6,571 organismos. Los nitratos y los fosfatos también fueron importantes en los niveles inferiores de la estructura jerárquica del análisis RT, ya que al sumarse estos nutrientes con valores altos de $\mathrm{MO}(>1,07786)$ se presentó la mayor abundancia en el número de individuos (11,330).

Tabla 8. Comparación del índice gonádico mensual de Meoma ventricosa grandis entre estaciones (E1: La Playa, E2: EI Jardín y E3: Palmitas). *Diferencia significativa $(\mathbf{P}<\mathbf{0 , 0 5})$ / Monthly comparison of gonadal index of M eoma ventricosa grandis between stations (E1: La Playa, E2: El Jardín and E3: Palmitas). *Significative difference $(P<0.05)$

\begin{tabular}{lrrrrrl}
\hline \multirow{2}{*}{ Mes } & \multirow{2}{*}{$\begin{array}{c}\text { Suma de } \\
\text { cuadrados }\end{array}$} & $\begin{array}{c}\text { Cuadrado } \\
\text { medio }\end{array}$ & $\mathrm{F}$ & \multicolumn{3}{c}{$P$} \\
\cline { 5 - 7 } & & & & E1 y E2 & E1 y E3 & E2 y E3 \\
\hline & & & & & & \\
Abril 06 & 46,212 & 23,106 & 12,587 & 0,4083 & $0,0023^{*}$ & $0,0005^{*}$ \\
Mayo 06 & 18,408 & 9,204 & 5,415 & 0,4475 & $0,0351^{*}$ & $0,0082^{*}$ \\
Julio 06 & 39,920 & 19,960 & 15,871 & 0,3930 & $0,0002^{*}$ & $0,0017^{*}$ \\
Septiembre 06 & 13,161 & 6,580 & 6,019 & 0,3839 & $0,0103^{*}$ & $0,0353^{*}$ \\
Octubre 06 & 0,178 & 0,089 & 1,922 & 0,0963 & 0,8898 & 0,3454 \\
Noviembre 06 & 8,436 & 4,218 & 15,205 & 0,6973 & $0,0004^{*}$ & $0,0012^{*}$ \\
Enero 07 & 7,847 & 3,924 & 4,879 & 0,6691 & $0,0182^{*}$ & $0,0246^{*}$ \\
Febrero 07 & 4,469 & 2,235 & 21,246 & $<0,0001$ & $0,0016^{*}$ & $0,0345^{*}$ \\
Marzo 07 & 4,482 & 2,241 & 2,092 & 0,0682 & 0,5106 & 0,2457 \\
& & & & & & \\
\hline
\end{tabular}




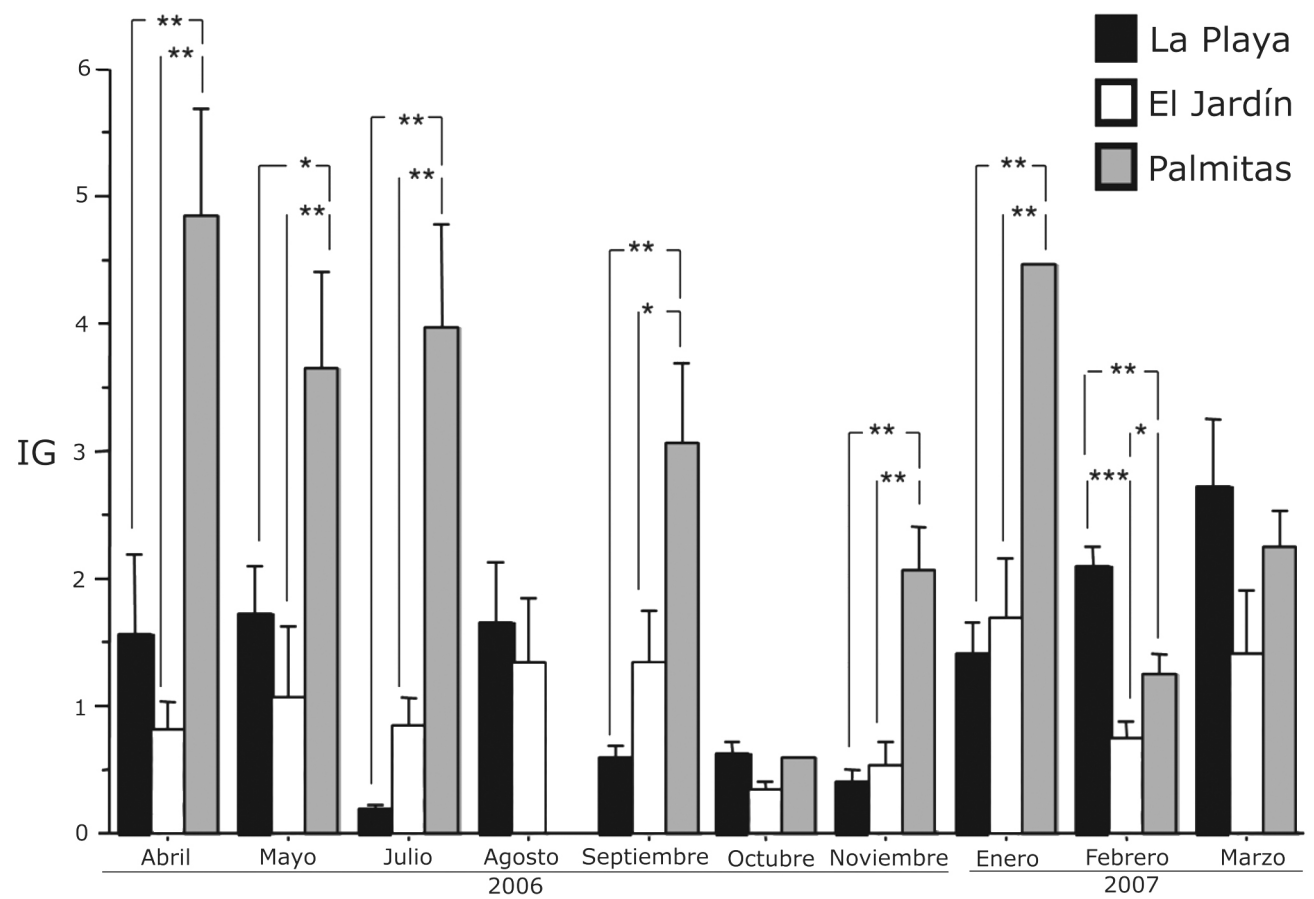

Figura 2. Índice gonádico (IG) promedio de individuos entre estaciones (La Playa, El Jardín y Palmitas) por mes de 2006 a 2007. $* \mathrm{P}<0,05 * * \mathrm{P}<0,025 * * * \mathrm{P}<0,001$. IG=índice gonádico / Average of gonadal index (IG) of individuals between stations (La Playa, El Jardín, and Palmitas) by month of 2006 to 2007. ${ }^{* P}<0.05 * * P<0.025 * * * P<0.001$. IG = gonadal index

\section{DisCUSIÓN}

Los datos de tamaño corporal de cada uno de los individuos de Meoma ventricosa grandis del canal Boca Chica, Acapulco, señalaron una diferencia entre las tallas de los organismos que formaban cada uno de los parches poblacionales. Las estaciones La Playa (1) y El Jardín (2) presentaron un porcentaje mayor de individuos juveniles sobre los adultos, en una proporción de 7:1 y 19:1, respectivamente. Ambas zonas se caracterizan por depósitos de bioclastos grandes, arenas muy gruesas y abundancia de gravas $\left(\mathrm{M}_{\mathrm{z}}(\phi)=\right.$ $-1,12 \pm 0,56$ y $-0,93 \pm 0,63)$, asimétricas $\left(\mathrm{Sk}_{\mathrm{I}}=-0,492 \pm\right.$ $1,815$ y $-1,132 \pm 5,735)$ y de tamaños diversos $\left(\sigma_{\mathrm{I}}(\phi)=0,795\right.$ $\pm 0,288$ y $0,838 \pm 0,300$ ), con una profundidad del sedimento de 20 a 30 cm. La estación Palmitas (3) se distinguió por un número semejante de organismos juveniles y adultos, en una proporción aproximada de 1:1, predominando los juveniles de tallas mayores a $70 \mathrm{~mm}$ de longitud. Esta estación se caracterizó por sedimentos de arena muy gruesa $\left(\mathrm{M}_{\mathrm{Z}}(\phi)=-0,63 \pm 0,19\right)$, de tamaños similares $\left(\sigma_{\mathrm{I}}(\phi)=0,474 \pm 0,077\right)$, casi simétricos $\left(\mathrm{Sk}_{\mathrm{I}}=0,110 \pm 0,099\right)$ y con una profundidad de la capa de sedimentos de $50 \mathrm{~cm}$. En todas las estaciones los ejemplares juveniles estaban enterrados entre los 10 y $20 \mathrm{~cm}$ de profundidad, mientras que los adultos se encontraron entre los 15 y $40 \mathrm{~cm}$. El análisis de varianza señaló que los individuos de mayor tamaño se encontraban principalmente en el parche poblacional de la estación 3 , con una diferencia significativa a las otras dos estaciones $(>0,001)$. Esta diferencia de tamaños pudo relacionarse con el IG, el cuál presentó valores significativamente mayores en los ejemplares de Palmitas (estación 3).

El análisis de la distribución de tamaños de acuerdo a la profundidad del sustrato muestra que la estructura de tallas corporales de $M$. ventricosa grandis se encuentra directamente relacionada con la textura y profundidad del sedimento, y se puede relacionar con las consideraciones descritas por Walker \& Gagnon (2014) que postulan que en erizos irregulares la eficiencia y velocidad de enterramiento está determinada por el tamaño de sus espinas, es decir, plantean que los individuos pequeños con espinas cortas se enterrarán especialmente en sedimentos con poca profundidad, donde exista una 


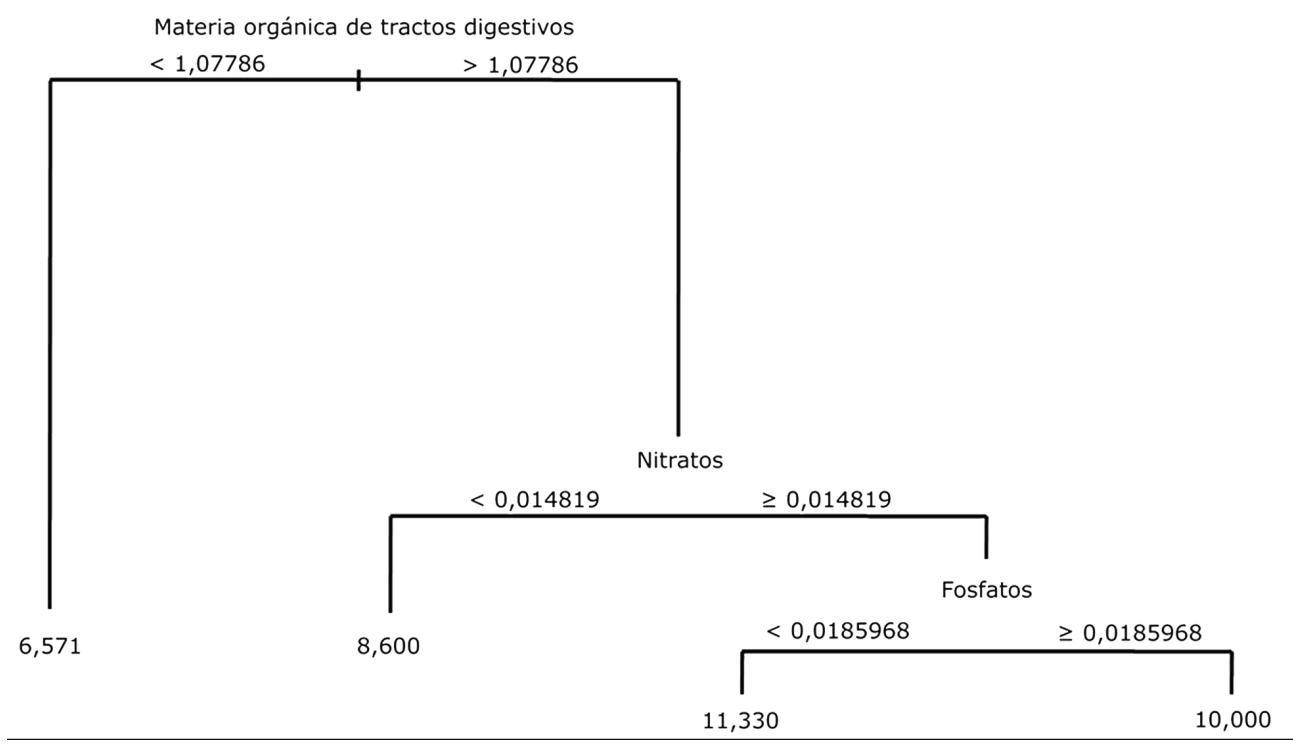

Figura 3. Análisis de árboles de regresión (RT): Individuos de las 3 estaciones (La Playa, El Jardín y Palmitas) (variable dependiente) contra las diferentes variables independientes (temperatura, salinidad, oxígeno disuelto, turbidez, nitrito, nitrato, amonio, fosfato, MO de nieve marina, MO del sedimento, MO del tracto digestivo) del canal Boca Chica, Acapulco, México de marzo 2006 a marzo 2007 / Regression Trees analysis (RT): Individuals of the 3 stations (La Playa, El Jardín and Palmitas) (dependent variable) versus the different independent variables (temperature, salinity, dissolved oxygen, turbidity, nitrite, nitrate, ammonium, phosphate, OM of marine snow, OM of sediment, OM of digestive tract) from Boca Chica Channel, Acapulco, Mexico, March 2006 to March 2007

predominancia de clastos grandes, gruesos y angulosos; en contraste, los especímenes de mayor tamaño con espinas largas habitarán principalmente en sedimentos con clastos más finos y trabajados, donde la profundidad del sustrato sea mayor y se puedan desplazar con mayor facilidad.

Previamente se ha postulado que los erizos irregulares no presentan selectividad por un sedimento en especial, ya que al ser detritívoros activos se pueden alimentar constantemente de diferentes partículas con el tamaño adecuado para poder ser ingeridas (De Ridder et al. 1987, Challener et al. 2009, Gauthier et al. 2011), sin embargo, en esta investigación se detecta por primera vez en Meoma la existencia de una selección específica del tipo de sustrato, dependiente principalmente de su talla corporal. Esta selección también se observa en otros invertebrados infaunales, los cuales seleccionan el sustrato en función a su tamaño y peso corporal (Pinedo $e t$ al. 2000, Robson et al. 2005).

Analizando las variables fisicoquímicas presentes en el canal se corroboró que sufrieron de sutiles modificaciones continuas entre estaciones, destacando la moderada variabilidad en la concentración de nutrientes sobre los factores físicos. En particular la cantidad de MO como nieve marina depositada en el área de estudio se mantuvo en un porcentaje de medianamente pobre $(1,84 \pm 0,73)$ a extremadamente rico $(3,60 \pm 1,69)$, reflejando un buen aporte del recurso sobre la región. En contraste, la MO del sedimento reflejó cantidades inferiores, manteniéndose de extremadamente pobre $(0,423 \pm$ $0,218)$ a pobre $(0,655 \pm 0,272)$; sugiriendo una disminución en la cantidad de materia conservada en el sustrato a causa de los diferentes mecanismos de degradación de las partículas (Kiørboe 2001). Por otro lado, la MO presente en los tractos digestivos tuvo un porcentaje sostenido en todos los individuos, de pobre $(1,50 \pm 0,85)$ a mediano $(1,94 \pm 0,78)$, señalando una alimentación continua de $M$. ventricosa grandis sobre el recurso en todas las estaciones.

Al reunir los resultados de las diferentes variables fisicoquímicas y relacionarlos con los datos de cantidad de individuos, se pudo identificar mediante el análisis RT que el porcentaje de $\mathrm{MO}$ y el aporte de nutrientes como nitratos y fosfatos actúan como las variables más importantes para que M. ventricosa grandis se pueda distribuir dentro del canal, 
favoreciendo su presencia en la región por la combinación de estos recursos en particular.

En conclusión, el análisis de varianza prueba que las poblaciones de Meoma ventricosa grandis presentes en las estaciones de estudio del canal Boca Chica muestran proporciones diferentes en la abundancia relativa de las tallas de los individuos.

Entre las 3 estaciones, las diferencias sedimentológicas más notables fueron la mayor profundidad de la capa de sedimentos, la buena clasificación y menor tamaño de los clastos que se encuentran en la estación Palmitas. Los resultados obtenidos permitieron registrar la selectividad específica de Meoma ventricosa grandis a un tipo de sustrato dependiendo principalmente de su talla corporal.

El estudio del IG señaló que $M$. ventricosa grandis presenta un ciclo reproductivo de tipo asincrónico.

Los resultados que se obtuvieron de los análisis de las variables fisicoquímicas y su relación con la abundancia del equinoideo estudiado (análisis RT) señalan que el porcentaje de MO del medio y el aporte de nitratos y fosfatos actúan como las variables más importantes que favorecen la presencia y estacionalidad de $M$. ventricosa grandis en el canal Boca Chica, Acapulco, México.

\section{Agradecimientos}

Los autores agradecen el apoyo de A. Gracia-Gasca, Director del ICML, UNAM cuando se llevó a cabo este estudio; a A. Duran por su ayuda técnica, a F. Vázquez-Gutiérrez por su asistencia en el análisis fisicoquímico, a B.P. Serrano-Flores por su ayuda en el análisis estadístico y al Equipo de Buceo de la Facultad de Ciencias, UNAM por su apoyo en el trabajo de campo.

\section{LITERATURA CITADA}

Aslan-Cihangir H \& MA Pancucci-Papadopoulou. 2012. Spatial and temporal variation of echinoderm assemblages from soft bottoms of the Çanakkale Strait (Turkish Strait System) with a taxonomic key of the genus Amphiura (Echinodermata: Ophiuroidea). Turkish Journal of Zoology 36(2): 147-161.

Barber DG, NR Gobius, IJC Hannah \& DP Poppi. 2001. The use of regression tree analysis to identify interactions between on-farm factors affecting milk protein content. Animal Production in Australia 25: 214.

Boos K, L Gutow, R Mundry \& H Franke. 2010. Sediment preference and burrowing behavior in the sympatric brittlestars Ophiura albida Forbes, 1839 and Ophiura ophiura (Linnaeus, 1758) (Ophiuroidea, Echinodermata). Journal of Experimental Marine Biology and Ecology 393: 176-181.
Buitrón-Sánchez BE, A Laguarda-Figueras \& FA SolísMarín. 1999. New record of Meoma ventricosa grandis Gray, 1851 (Echinodermata, Echinoidea: Brissidae) from the Lower Pliocene of Baja California, Mexico. Coloquios de Paleontología 50: 151-160.

Challener RC, MF Miller, DJ Furbish \& J McClintock. 2009. Evaluation of sand grain crushing in the sand dollar Mellita tenuis (Echinoidea: Echinodermata). Aquatic Biology 7:261-268.

De Ridder C, M Jangoux \& L De Vos. 1987. Frontal ambulacral and peribuccal areas of the spatangoid echinoid Echinocardium cordatum (Echinodermata): a functional entity in feeding mechanism. Marine Biology 94: 613-624.

Díaz JF \& JC Correa. 2013. Comparación entre árboles de regresión CART y regresión lineal. Comunicaciones en Estadística 6(2): 175-195.

Díaz NA, JA Bárcena, E Fernández, A Galván, J Jorrín, J Peinado, FT Meléndez-Valdés \& I Túnez. 2000. Espectrofotometría: espectros de absorción y cuantificación colorimétrica de biomoléculas. Departamento de Bioquímica y Biología Molecular, Campus Universitario de Rabanales, Facultad de Medicina, Córdoba, pp. 1-8.

Folk RL. 1980. Petrology of sedimentary rocks, 190 pp. Hemphill Publishing Company, Austin.

Freeman SM \& SI Rogers. 2003. A new analytical approach to the characterization of macro-epibenthic habitats: linking species to the environment. Estuarine, Coastal and Shelf Science 56: 749-764.

Garay J, G Ramírez, JM Betancourt, B Marín, B Cadavid, L Panizzo, L Lesmes, JE Sánchez, SH Lozano \& A Franco. 2003. Manual de técnicas analíticas para la determinación de parámetros fisicoquímicos y contaminantes marinos: agua, sedimentos y organismos. Serie Documentos Generales 13: 1-117. Instituto de Investigaciones Marinas y Costeras-INVEMAR, Santa Marta.

Gauthier R, A Ziegler \& C De Ridder. 2011. Presence of a seawater-lled caecum in Echinocardium cordatum (Echinoidea: Spatangoida). Journal of the Marine Biological Association of the United Kingdom 92(2): 379-385.

Gray JS. 2002. Species richness of marine soft sediments. Marine Ecology Progress Series 244: 285-297.

Guzmán HM \& CA Guevara. 2002. Annual reproductive cycle, spatial distribution, abundance, and size structure of Oreaster reticulatus (Echinodermata: Asteroidea) in Bocas del Toro, Panama. Marine Biology 141: 1077-1084.

INECC. 2009. Manual de procedimientos estándares para el análisis histológico e histopatológico en organismos acuáticos, 22 pp. Universidad Autónoma de México-Instituto Nacional de Ecología. México D.F.

Iken K, T Brey, U Wand, J Voigt \& P Junghans. 2001. Food web structure of the benthic community at the Porcupine Abyssal Plain (NE Atlantic): a stable isotope analysis. Progress in Oceanography 50: 383-405. 
Kiørboe T. 2001. Formation and fate of marine snow: smallscale processes with large-scale implications. Scientia Marina 65(2): 57-71.

Méndez-Ubach N, V Solís-Weiss \& A Carranza-Edwards. 1986. La importancia de la granulometría en la distribución de los organismos bentónicos. Estudio de playas del Estado de Veracruz, México. Anales del Centro de Ciencias del Mar y Limnología, Universidad Nacional Autónoma de México 13(3): 45-55.

Moreno DR. 1978. Clasificación de pH del suelo, contenido de sales y nutrimentos asimilables, $180 \mathrm{pp}$. Instituto Nacional de Investigación Agropecuaria-Secretaría de Agricultura y Recursos Hidráulicos, México, D.F.

Mortensen Th. 1951. A monograph of the Echinoidea, Vol. 2, Spatangoida II, 593 pp. C. A. Reitzel Publisher, Copenhagen.

Pinedo S, R Sardá, C Rey \& M Bhaud. 2000. Effect of sediment particle size on recruitment of Owenia fusiformis in the Bay of Blanes (NW Mediterranean Sea): an experimental approach to explain field distribution. Marine Ecology Progress Series 203: 205-213.

Pulliam HR. 2000. On the relationship between niche and distribution. Ecology Letters 3: 349-361.

Ramírez-Llodra E, PA Tyler \& DSM Billett. 2002. Reproductive biology of porcellanasterid asteroids from three abyssal sites in the northeast Atlantic with contrasting food input. Marine Biology 140: 773-788.

Robson BJ, LA Barmuta \& PG Fairweather. 2005. Methodological and conceptual issues in the search for a relationship between animal body-size distributions and benthic habitat architecture. Marine and Freshwater Research 56: 1-11.

Snelgrove PVR \& CA Butman. 1994. Animal-sediment relationship revisited: cause versus effect. Oceanography and Marine Biology: an Annual Review 32: 111-177.

Solís-Marín FA, A Laguarda-Figueras \& A Leija-Tristán. 1997. Morphology, systematics and distribution of Meoma ventricosa grandis and $M$. ventricosa ventricosa (Echinodermata: Echinoidea: Brissidae) along Mexican Coasts. Proceedings of the Biological Society of Washington 110(2):301-309.
Solís-Marín FA, MBI Honey-Escandón, MD HerreroPerezrul, F Benitez-Villalobos, JP Díaz-Martínez, BE Buitrón-Sánchez, JS Palleiro-Nayar \& A DuránGonzález. 2013. The echinoderms of Mexico: biodiversity, distribution and current state of knowledge. In: Alvarado JJ \& FA Solís-Marín (eds). Echinoderm research and diversity in Latin America, pp. 11-66. Springer-Verlag, Berlin Heidelberg.

Torres-Martínez MA. 2008. Amplitud de nicho alimentario, utilización de sedimento, reproducción y distribución de Meoma ventricosa grandis Gray 1851, (Echinodermata: Brissidae) en el canal Boca Chica, Acapulco, Guerrero, México. Tesis de Maestría, Instituto de Ciencias del Mar y Limnología, Universidad Nacional Autónoma de México, México, 76 pp.

Torres-Martínez MA \& FA Solís-Marín. 2010. Sediment utilization and feeding-niche breadth of Meoma ventricosa grandis (Echinodermata: Brissidae) at Boca Chica Channel, Acapulco, Guerrero, Mexico. In: Harris LG, SA Böttger, CW Walker \& MP Lesser (eds). Echinoderms: Durham. Proceedings, pp. 643-645. CRC Press, Taylor and Francis Group, Balkema, Leiden.

Torres-Martínez MA, FA Solís-Marín, A LaguardaFigueras \& BE Buitrón-Sánchez. 2008. Poros filodiales en la identificación de dos subespecies de erizos de mar: Meoma ventricosa grandis (Océano Pacifico) y Meoma ventricosa ventricosa (Océano Atlántico). Revista de Biología Tropical 56(3): 13-17.

Walker DE \& JM Gagnon. 2014. Locomotion and functional spine morphology of the heart urchin Brisaster fragilis, with comparisons to B. latifrons. Journal of Marine Biology 2014: ID 297631, <dx.doi.org/10.1155/2014/297631>, 9 pages.

Williamson JE \& PD Steinberg. 2002. Reproductive cycle of the sea urchin Holopneustes purpurascens (Temnopleuridae: Echinodermata). Marine Biology 140: 519532

Recibido el 5 de marzo del 2015 y aceptado el 4 de mayo del 2016

Editor: Claudia Bustos D. 